Article

\title{
Exploring the Ways Cinematography Affects Viewers' Perceived Empathy towards Onscreen Characters
}

ELEN LOTMAN, Tallinn University Baltic Film, Media, Arts and Communication School, Estonia; email: elen.lotman@tlu.ee 


\section{ABSTRACT}

In the history of cinematography there is a noticeable tradition to deliberately highlight the elements that accentuate space and spatiality in the shots. At the same time, there is also a contrary tradition, i.e. the conscious reduction of spatiality with the help of artistic tools in order to evoke a feeling of alienation. In this article I will argue that it is highly likely that the visual reinforcement of depth has become one of a cinematographer's most frequently used tools, because it plays an important role in the audience's perceived empathy towards onscreen characters. Since the practices of art-making - e.g. cinematography - represent a way that the empirical experience accumulated in professional practices reflects underlying neural processes, this article will first draw upon evidence from the common tenets of cinematography and reflect on how these correspond to the respective phenomena in human perception and cognition. The second part of the article examines the theory of the para-dramatic and eso-dramatic factors established by Gal Raz and Talma Hendler as it applies to cinematography; thereby suggesting possibilities for broadening the theoretical foundations of the twofold division of the causes for the viewers' empathetic responses. The article will also introduce the results from a pilot experiment. However, I will not argue that the rendering of cinematographic space and drawing attention to certain areas are superior tools for creating filmic empathy. I will rather point out that they are often used by cinematographers when they want to create an immersive experience, and therefore, there is reason to believe that a connection exists between emotional empathy and the usage of these cinematographic tools. 


\section{INTRODUCTION}

\subsection{Clarifying the terminology}

Because this article is an interdisciplinary endeavour, the author, who is a professional cinematographer, is embarking on research that uses terminology from both practical filmmaking and empirical research fields. Since some of the terms used in this article are often used in different contexts, the meanings of the most central ones need some clarification within the context of this article.

\section{Cinematography}

In this article, the term cinematography refers to everything connected to camerawork (camera movement, lighting, lenses etc.), not the wider use of the same term, which in theoretical literature, sometimes refers to the elements of film form more generally. In different theoretical works, the word 'cinematography' has often been given a far broader definition than the one used by filmmakers.

\section{Empathy}

Empathy research is a growing field with an abundance of literature available in fields ranging from philosophy to neuroscience and psychology. A recent review by Jamil Zaki and Kevin N. Ochsner (2012) defines three broad classes for empathic processes, for which different terminology is often used:

(a) experience sharing (related terms include affective empathy, emotional contagion, embodied simulation): an affective response to another person often entails sharing that person's emotional state (i.e. empathic distress or resonance);

(b) mentalising (related terms include cognitive empathy, Theory of Mind): a cognitive capacity to assume the perspective of the other person (i.e. sympathy, compassion);

(c) and prosocial concern: the motivation to help others that can be based on (a), (b) or both.
Prosocial motivation has recently been receiving systematic attention and film scholars often use a twofold division of empathetic reactions, including approaches related to mentalising and experience sharing, or, more specifically, the Theory of Mind (ToM) and Embodied Simulation (ES) (see Raz, Hendler 2014 for a recent review). Although the issue of how humans feel empathy have been debated philosophically long before neuroscientists were actually able to track its neurological basis, current research supports the concept of two different neural systems - one is more bodily and emotion-driven, the other is more cognitive. However, it should be noted that while these two processes have previously been often studied separately, a growing body of evidence shows that empathic reactions can be fully understood when both systems are viewed in combination (Zaki, Ochsner 2012 and references therein).

Gal Raz and Talma Hendler's article goes further in the division of empathic reactions to film, and initiates comparative research in order to find the responses in the brains of film audiences that correspond to these two empathetic states. While viewing two clips from Stepmom (USA, 1998, directed by Chris Columbus, cinematographer Donald M. McAlpine) and Sophie's Choice (USA, 1982, directed by Alan J. Pakula, cinematographer Nestor Almendros) two different viewer responses were described, which correspond to the ToM and ES circuits, and therefore a system of paraand eso-dramatic factors of the film form can be derived from these results.

\subsection{Cinematography as the art of space - cinematographic canons related to rendering the depth of the shot}

However, before this division can be explained we need to take an in-depth look at the canons of cinematography, which have developed based more on artistic practices, than on scientific research. It has been pointed out that certain elements have been tested through trial and error during the century-long history of film 
language, and they have found their way into the artistic canons of the language itself, for example, in editing:

The more probable devices became the standard practices of the classical editing system because they fell within the accommodation ranges of the cognitive and perceptual processes required for perceptual continuity, and therefore, have been handed down through apprenticeship, film schools, production handbooks, and film studies textbooks. (Berliner, Cohen 2011: 48)

The same thing seems to have happened in cinematography during the 100-plus years of the cinema. There are certain rules of the craft that are handed down from one generation of cinematographers to the next (i.e. the so-called key to fill lighting ratio, lighting for depth etc.) without much explanation of how these rules developed or why. If we take a deeper look at these rules, we see a clear reinforcement of cognitive and perceptual processes through the use of different cinematographic tools. Many of these tools are directly connected to rendering depth and re-enforcing the sense of depth through monocular depth cues. This article is also based on the observation that there are cinematographic conventions in terms of lighting, movement and framing that impact the sense of space of the audience and these can be traced through film history. If we take a look at any of the wellknown cinematography handbooks, we can find instructions on how to do this:

As we discussed in Shooting Methods, one of the key elements of film is that we are projecting 3-dimensional space onto a 2-dimensional plane. Except where we want this flatness, it is a goal to recreate the depths that existed in the scene. A big part of this is to create shots with a foreground, mid-ground, and background. [---] Light has a great power to form space. In this case, the single source forms a pool of space that envelops the students. Outside it is another space, sharply delineated.

(Brown 2013:31)

In order to map out the possibilities for an interdisciplinary study of cinematography through film theory and the cognitive sciences, we can take a look into how the most influential cinematographers explain their work and how this has translated into the internal teachings of cinematography. It is clear that none of them was a neuroscientist and therefore did not possess the relevant neuroscientific information needed to base the artistic decisions thereon. However, what is a cinematographer, besides being the person behind the camera and the artistic partner of the team leader? She or he is a person very well trained in seeing. And it seems that the sense of what is 'right' or 'well composed' is inherently connected to the cinematographer's perception. Thus, through the cinematographer's well-trained vision as a medium, the human brain's preferences towards what makes it feel more immersed or alienated translates into the artistic choices made by the cinematographer. Vittorio Storaro, one of the great cinematographers of our times, whose work has immensely influenced both audiences and the canons of professional cinematography, and whose body of work is routinely researched, seems to have worked a lot on the space, as the analysis of his work often shows. For example,

Storaro suggests that movement on screen is about space, since it bridges the gap between the two tenses of photography - here and now; there and then - while it also connects the empty figures on the screen and the full bodies in the audience. (Dalle Vacche 2009) 
In Angela Dalle Vacche's article the term chiaroscuro is also mentioned. So-called chiaroscuro lighting, made famous by Renaissance painters, refers to the use of strong contrasts between light and dark in representational art, in order to recreate the sense of volume in objects and figures. The term has found its way into photography and cinematography, and evolved into 'checkerboard lighting'. (Figure 1) This technique is based on positioning light areas against dark areas in the shot and vice versa in order to recreate a sense of depth (this reinforces a monocular depth cue known as occlusion). These techniques started to be commonly learned when studying cinematography and became established as rules. For example, the Provideo Coalition, a well-known tutorial site on cinematography, describes why one should use checkerboard pattern lighting as follows:

You see how this creates depth in your scene and it gives you enough of the background to let the audience know the setting and set the mood but not so much that it distracts you from the talent and where the director wants the audience to focus their attention. (Marko 2014)

Recreating the sense of depth is something that has been regarded as one of the most important techniques used by cinematographers. A sense of depth is a complex perceptual process that arises from different perceptual cues, including oculomotor, binocular and monocular depth cues; the latter being the only one that is available to classic 2D cinematographers. In web tutorials - such as Nofilmschool, a very well established contemporary teaching and learning tool - one can find numerous clips and articles that introduce the notion of monocular depth cues to the cinematography crowd. In its description

1 Examples can be found here: http://nofilmschool.com/ 2014/12/5-techniques-create-depth-make-

cinematography-more-dynamic; here: of the cinematography in James Bond movie Skyfall (UK/USA, 2012, directed by Sam Mendes, cinematographer Roger Deakins), the same source also states,

The other reason that practicals are an incredibly powerful tool for cinematographers is that they're a simple and effective way to create depth in a scene. Of course, depth can be achieved in any number of ways, but lighting is one of the best. Placing practical lights at various depths throughout a scene can not only help you motivate any artificial sources you may want to use, but they can drastically enhance the way the audience perceives spatial depth. (Hardy 2015)

Many of the elements listed in these articles have been a part of the history of cinematography for a long time; for example, backlighting has been used as a tool to separate an object from the background since the era of black-and-white films. The neurophysiological correlate to this could be a process called segregation, which is one of the basic stages of object recognition:

Normally, in order for something to be identified and represented as a figure, its contours need to be identified. Therefore, figureground segregation is intimately associated with the presence or at least perception of contours. (Ferber et al. 2005)

Another well-known lighting canon is the 'pools of light' method, whereby pools of light act as a texture gradient type of monocular cue, and the receding pools of light reinforce the sense of depth. (Figure

2 and 3) We can find a reference to it on the website Dvxuser, which states that

http://nofilmschool.com/2015/11/lighting-likevermeer-create-cinematic-depth-lighting; and here: http://nofilmschool.com/2013/07/video-add-depthto-your-shots. 

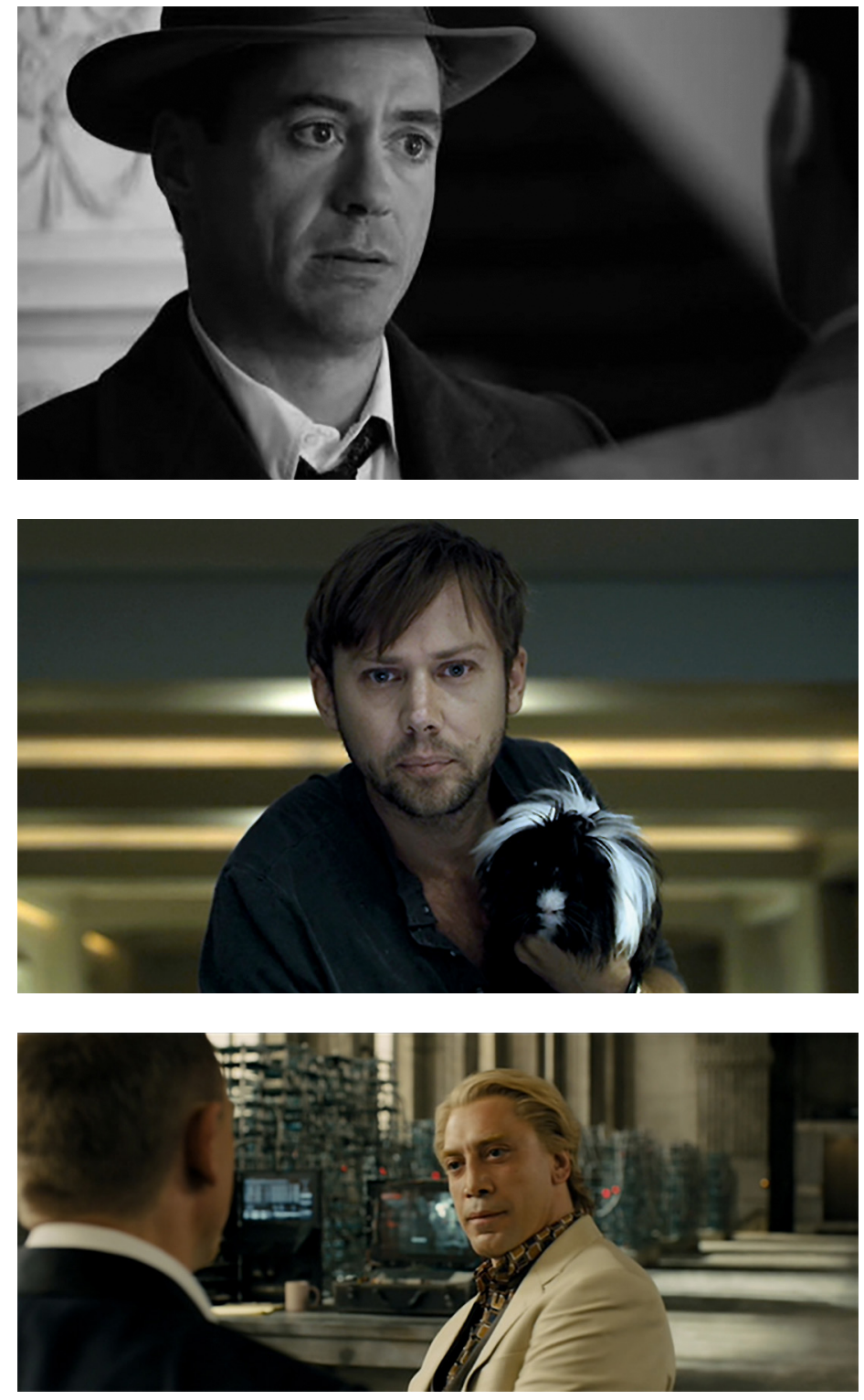

FIGURE 1. Good Night, and Good Luck (USA/France/UK/Japan, 2005, directed by George Clooney, cinematographer Robert Elswit).

An example of 'checkerboard lighting' in a portrait shot, with 'short key' or 'far side key' lighting combined with background lighting, forming a light-on-dark and dark-on-light pattern that reinforces the sense of depth due to a monocular cue of occlusion.

FIGURE 2. House of Cards (USA, 2013-present).

FIGURE 3. Skyfall.

Examples of 'pools of light' lighting method working in the background of portrait close-ups and creating a monocular depth cue of texture gradient. 
Pools of light are an excellent example of light defining space. Pools of light selectively highlight certain areas of a space, defining a 'here' and 'there' and helping create a spatial relationship between them and thus of the scene. (O'Hara 2010)

The same source describes 'lighting in layers', a method similar to the aforementioned 'checkerboard lighting' and the author describes the reason for using this method as following:

By establishing visual reference
points within a scene, such as 'it's
light here, but dark over there', the
cinematographer can enhance
the illusion of depth as the light
is now defining distance, rela-
tive positions, and dimensions of
the physical space. These aides
help the human brain to create
an idea of the space in three-
dimensional terms, although only
seeing a two-dimensional image.
[---] Within the 'lighting in layers'
approach, it is often the practice
to darken the foreground and
background elements in com-
parison to the lit subject, which
usually falls somewhere between
the two. This lighting technique
not only establishes the light as
being 'here and not there', but
creates subject-background
separation as well. The visual
contrast between the lit subject
and darker background results
in an illusion where the subject
will appear to 'pop' away from the
background. (O'Hara 2010 )

In portrait lighting there is a method called 'short lighting' or 'far-side key', in which the main light is positioned on the other side of the subject - the subject's viewpoint axis from the camera - thus rendering the volume and the shape of the face. 'Short lighting' is often used in combination with 'checkerboard lighting' in order to create a maximum sense of depth for the audience's sense of the place within the scene. Shutter Angle, another popular resource among cinematographers, presents the depth obsession in cinematography in many of its tutorials; for example, a three-part article on how to create space, which lists most of the best-known techniques used by directors of photography. In explaining why one needs to recreate depth, the article points out:

Depth is not a universal quality to look for in an image, but most non-abstract images benefit from an enhanced depth illusion. After all, an image renders a reality (existent or imagined), and reality is $3 \mathrm{D}$. A cinematic 2D representation should appear sufficiently three-dimensional. Depth defines space. Injecting depth into an image furthers the spatial awareness of the viewer and helps orient them into the depicted world. Ideally, the objects in the frame should appear recessing behind the screen. (Shutter Angle 2012)

As shown above, cinematographers use a lot of tools of the trade to tap into our perceptual processes and recreate a sense of space and depth inside the audience's brains. But why is it so important that it has almost become a historic obsession within the art of cinematography itself?

\subsection{Perception of space and its possible connection to empathy}

If we look at the recent findings about human and primate brain function, we will find apparent connections between the perception of space and emotional reactions to presented stimuli.

In an article on how the human amygdala encodes value and space during decision-making, Olga Therese Ousdal et al. (2014) describe the amygdala as the key structure that contains the value of sensory stimuli. They refer to a non-human primate 
study where the initial evidence showed that the amygdala also acts to merge value with spatial location. The experiments with humans described in Ousdal et al. (2014) show that the amygdala provides the representation of space and value that play an important role in the spatial attention of highly valuable stimuli, since subjects responded faster and with more amygdala activity when the reward depended on spatial specificity. Other experiments with non-human primates also suggest that although the amygdala has long been considered an essential coordinator of emotion, it is possible that it also plays a major role in exhibiting emotionally important cues within space (Ousdal et al. 2014). When it comes to humans, Ousdal et al. (2014) raise questions about the connections between a stimulus's value and its spatial location, hypothesising that the two converge in the human amygdala.

Various experiments have shown that connection between the sense of space and emotional empathy can be traced inside the amygdala, since the amygdala, as a part of the brain that modulates space perception, also plays a crucial role in the feeling of emotional empathy. Obviously, amygdala activation is connected to the other parts of the brain and the amygdala is not solely responsible for the creation of emotional empathy, but there is strong evidence that it does play a role. To be more precise, Kevin C. Bickart et al. (2014) argue that a 'growing body of evidence suggests that the amygdala is central to handling the demands of complex social life in primates' (Bickart et al. 2014).

The development of our social life and embodied simulation are strongly interconnected, as shown by Vittorio Gallese (2009), indicating that the neural mechanisms underpinning embodied simulation enable the early development of different elements of our social life. If the amygdala plays an important role in the emotional and social life of humans in general, there is also proof that the regulation of emotional empathy itself is directly connected to the amygdala. A recent quantitative study (Hillis 2014), which included several meta-analyses, identified activation in the right amygdala in connection with empathy. Another functional MRI study described by the same source used the evaluation of the impact of intranasal vasopressin on the amygdala during the onset of empathy, discovering that it did modulate the right amygdala incitement that is associated with emotional empathy (Hillis 2014). The study indicates that amygdalae are involved in a variety of emotional processes and it has been shown that amygdala lesions negatively affect the performance of emotional empathy tasks, whether through emotional contagion (ES) or affective perspective-taking (ToM). In a review on how focal lesions affect emotional empathy, Argye E. Hillis (2014) concludes that the amygdala is part of the circuit that mediates the emergence of emotional contagion. There is also another study by René Hurlemann et al. (2010) that draws upon the evidence that the amygdala does not seem to influence cognitive empathy (ToM). On the basis of the research done on the effect of oxytocin on two patients with selective bilateral damage to the amygdala (monozygotic twins with congenital Urbach-Wiethe disease), it was determined that their cognitive empathy was not undermined, while their emotional empathy was compromised (Hurlemann et al. 2010). These findings show that there is a certain parallelism in brain circuits (e.g. the amygdala) regulating emotional empathy (Embodied Simulation) and the sense of space, while the emergence of cognitive empathy (Theory of Mind) does not seem to be a precondition for spatial valence evaluation. Obviously these studies do not prove a direct link between the perception of space and emotional empathy, but a certain functional overlap within the same neural circuits connected through the amygdala has been established. Thus there is good reason for planning further studies in this area, especially since film language has inherent possibilities that have yet to be explored by this research. Dolf Zillmann has drawn connections between viewing real-life situations and their cinematic 
representations in his account on audience reactions:

Reflexive and learned skeletalmotor reactions elicited by exposure to representations of high iconicity (i.e. photography, cinematography) that exhibit another person's manifest of impending emotional behavior are also subsumed in the dispositional component of empathy. (Zillmann 2013: 148)

The author also draws on the apparent realism of filmic images by stating that '[s] pecifically, iconic representations are considered capable of eliciting reflexive reactions because of great stimulus similarity with the represented events' (Zillmann 2013: 148).

Real perceived space has depth and we sense it by gathering depth information from all the available cues. If moving images with cinematographically reinforced depth are shown to audiences, creating a kind of super-depth, this could work as a kind of supernormal stimulus of reality (Ramachandran, Hirstein 1999).

Of course, there is also the important question of minimalist or abstract moving images, be they cartoons like Michaël Dudok de Wit's Father and Daughter (Netherlands, 2000) that clearly produce strong emotional reactions or Fritz Heider and Marianne Simmel's (1944) classic animation that was in itself an experimental study in apparent behaviour. If triangles can produce empathetic reactions in the audience, does realism even matter? This is of course an important subject, and it definitely requires future research, regardless of whether the empathy felt by the audience watching these more abstract images is the Embodied Simulation or Theory of Mind kind. However, for the case being presented in this article, there is ample proof that realism of display does play a crucial role in eliciting mirroring activities of the Embodied Simulation kind in the brain, e.g. watching the rigid movements of a robot's arm interferes less with viewer's own arm movement than watching human arm movements (Kilner et al. 2003). Or, consider the study by D. Perani et al. (2001) in which seeing real human hands in realistic environments activated the viewer's brain motor cortices, but similar actions by a geometrical virtual hand did not, engaging only prevalent visual perceptual processes. Finally, Shihui Han et al. (2005) observed motor-related brain activity when real humans were viewed on a video screen, but not in response to cartoon clips of human and non-human agents.

\subsection{The twofold division of cinematographic styles and its connection to cinematography}

Could it be that there is a strong connection between the ability to perceive space in a film world and to empathise with characters in that space? There is reason to believe that it might be true, as orientation in space is a crucial part of assessing the saliency of stimuli, and the more salient the stimuli, the easier it is to focus attention on important parts of the visual field in order to acquire information that enables the emergence of empathy. And this most likely applies to both real-life experiences and watching cinematically immersive images. One obvious explanation would be that the use of a camera makes the image more realistic to the audience, and thus we feel more empathy towards the characters. But this explanation is insufficient for two reasons.

In terms of cinematography, it is important to take in account that the realism felt by the audience has been created by the artistic choices of the cinematographer (since this article is concerned only with cinematography, and not acting, directing, editing or production design, we will not go into any detail on how these elements of film language create immersion and/or empathy).

Firstly, as all cinematographers and photographers know, realism is not something one can take for granted and is a priori a condition of merely using a camera to record images. A realistic painting can often feel more realistic than a photo or a 
filmed sequence - for reasons both physical (lenses etc.) and cognitive (mechanistic tools do not have the computing power that a painter's brain uses when making a painting more realistic while using brain processes like size constancy and colour constancy in the process). Thus, one could argue that if they wanted to, cinematographers could very easily make images look extremely unnatural and unrealistic with the same camera and lights that they use to make them look and feel realistic.

Secondly, if the mere fact of using the camera could create full immersive emotional empathy towards characters, could films that alienate the audience from the characters even exist? Obviously they could. There are probably almost as many films with the goal of immersing the audience as there are films with the exactly opposite goal - to constantly remind the audience that they are watching a film; or if not consciously reminding them of the artificial nature of the onscreen events, then at least not trying to completely immerse them in the characters.

An article about the limits of virtual reality (Sutherland 2016) describes this well-known clearly twofold distinction in the arts, whereby one approach strives for stronger immersion and the other has a goal of stronger alienation.

The word empathy derives from the German word, Einfühlung, which had been used in artistic contexts to describe the "feelinginto' of an object. This word acquired meaning as a way to characterize the embodied viewer response, drawing on both the fields of perceptual psychology and art history, an interest shared by the research communities of art and science. It rests on the idea that emotional identification is an internal, individual ability that can produce particular social feelings. It was also eventually rejected by dramatist Bertolt Brecht, whose participatory methods inspired Augusto Boal. Brecht believed that emotional identification ran counter to the ability of individuals to effect actual social change, because it lacked the moment of 'alienation' that could disrupt existing worldviews and provoke internal change. (Sutherland 2016)

In terms of cinematography, the latter could explain why Hollywood uses a special keyto-fill light contrast ratio for schadenfreude type comedies (laughing at others would usually require a more distanced stance towards the characters or more cognitive activity for self-recognition, because who wants to laugh at themselves?); on the other hand, dramas, thrillers or horror films tend to have high contrast ratios. Thus, the aforementioned cinematographic canons enhance the space perception of the audience by bringing along reinforced emotional empathy (except. of course, if the opposite is the goal). This would also explain why there are certain films that work against the canons of depth lighting and depth staging to create a Brechtian alienation, for example, the film worlds of Roy Andersson

(Figure 4) or Wes Anderson.

Based on this evidence one could hypothesise that there are some cinematographic tools that are used in films in order to reinforce embodied simulation though depth-emphasising cinematography (especially when corresponding to the typical needs of a genre, e.g. drama, thriller and horror, which benefits from the immersion effect). There are also some cinematographic tools that can be used in films where an alienation effect is desired to reinforce the Theory of Mind processes instead of Embodied Simulation ('Brechtian' films). And others in which rather than an alienation effect, less Embodied Simulation and more Theory of Mind is required in order for the genre to have the desired impact on the audiences (e.g. typical comedies).

And this is where the eso- and paradramatic factors come in. Raz and Hendler (2014) explain in their article that the 
selected film clip from Sophie's Choice recreated more of an eso-dramatic response, and the one from Stepmom more of a para-dramatic response. The hypotheses presented by the authors as to the reasons for these differences (e.g. in one clip the tragic event is happening in the present, and this makes it more physically immediate; and in the other it is a tragic event that will happen in the not so distant future, and therefore it is prone to evoke more cognitive reactions) are completely plausible. But there could also be another interpretation, which is probably not an alternative, but rather something that complements Raz and Hendler's approach (especially since the authors of the article have already listed some 'typical cinematic devices' in their division that respond to both types of dramatic factors). The cinematography of the clip from Stepmom (and much of the entire film for that matter) resembles a typical comedy more than a typical drama in the sense of film lighting canons, e.g. as mentioned above, the key-to-fill ratio that produces the contrast in this film is also rather common in comedies. Depth is less accented by the use of different depth cues and lighting (the key to fill light ratio is quite low), and the clip includes numerous long shots where characters are depicted in profile, thus lessening the sense of "being there' through eye line closeness. The character's eye line closeness to the camera axis is well suited to the list of the common devices for implying subjectivity, including shot types such as subjective shots, shot-reaction shots and eye line matches (Stam et al. 1992: 188). (Figure 5 and 6) However, the clip from Sophie's Choice is exactly the opposite: the use of high contrast lighting and backlighting reinforces the sense of depth in addition to the sense of drama. There are a lot of wide shots that emphasise the sense of depth through diagonal perspective lines and atmospheric haze (two monocular depth cues used often in immersive cinema), especially in the shots where the daughter of the main character, who is being taken away from the mother, distances from her crying - a tragic moment that seems to last forever, an effect fully empowered by both editorial (cross-cutting) and cinematographic (depth accenting) choices. (Figure 7 and 8) Both films serve as examples of the two different, and somewhat representative, types of cinematography that are mentioned above.

The authors probably did not select two clips that feature such an opposite use of cinematography intentionally, thus it could be quite incidentally that their article also provides proof on how the rendering of depth in cinematography has a different effect on the two types of empathy creation. This could be a very lucky accident for future research in cinematography and it most definitely lays the ground for possible further research.

\section{A PILOT STUDY}

In order to do a preliminary test on the hypothesis that space-emphasising cinematography has a stronger emotional impact on the audience, we designed and conducted a pilot experiment with the goal of later using the same stimulus and paradigm for the physiological measures of emotional affect.

The experiment was designed to research the impact of light on space perception, emotional arousal, as well as valence and subjective empathy. Two hypotheses that are related to depth and cinematography were established:

Hypothesis 1 - the reduction of the space inducing light will lead to reduced emotional reactions to facial expressions (empathic distress, connected with Embodied Simulation).

Hypothesis 2 - the reduction of the space inducing light will lead to increased cognitive reactions to facial expressions (compassion, connected with Theory of Mind).

\subsection{Methods}

\subsubsection{Participants}

Fifteen healthy females (between the ages of 26 and 52, with a mean age of 35) participated in the experiment. All the subjects gave their written informed consent to par- 

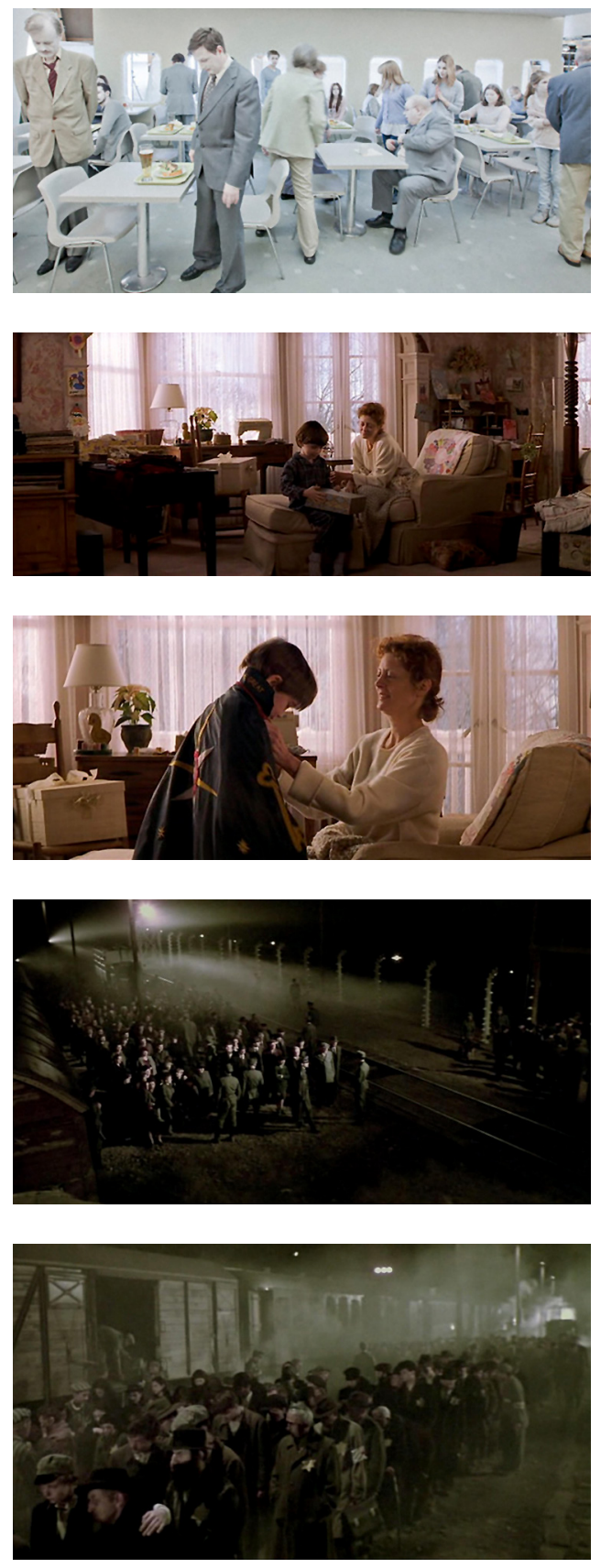

FIGURE 4. A Pigeon Sat on a Branch Reflecting on Existence

(En duva satt på en gren och funderade på tillvaron, Sweden/Norway/France/Germany, 2014, directed by Roy Andersson, cinematography István Borbás and Gergely Pálos).

An example of flat lighting and lack of monocular depth cues serving to create Brechtian alienation effect.

FIGURE 5 AND 6. Examples of high key lighting in Stepmom.

FIGURE 7 AND 8. Low key, contrast-enhancing lighting combined with monocular depth cues (relative size, perspective lines, receding contrast, elevation and texture gradient) in Sophies Choice. 
ticipate in the study, which was performed in accordance with the Declaration of Helsinki. The selection of only female subjects was motivated to avoid gender differences since the visual stimuli contained female actors.

\subsubsection{Stimuli}

A set of three typical lighting methods was chosen to create the visual stimuli; all of which induce a sense of space in portrait cinematography, as well as one method that did not directly affect the sense of space, but did draw attention to the eyes of the subject, thus strengthening the "popping out' effect in combination with the other lighting devices. These four methods were separable based on two light conditions, which are quite commonly used as 'sets' of lighting methods. Of course, it is possible for a cinematographer to choose only one or more of these lighting methods, but we decided that in combination they would produce the maximum effect, taking into account that all the other elements of film language that would otherwise enforce the emotional affect of the subjects (directing, set design, acting, dramaturgy, sound and music etc.) were missing in these shots.

The following four lighting methods for the portrait were used (the first representing a 'flat light' condition and the second representing a 'space-induced light' condition):

1. The separation of the objects from the background, variants: without backlight and with backlight.

2. Portrait lighting: so-called broad ('near side key') and short ('far side key') key light.

3. Background light: without and with.

4. Catchlight/eyelight: without and with.

In order for the stimulus to be compatible with the subsequent psychophysiological, and more specifically, facial electromyography (fEMG) testing, the videos were a priori designed to evoke maximum facial expression. In a study on the facial feedback hypothesis and its connections to emotional empathy (Andréasson 2010), the strength of the Embodied Simulation effect can be assessed by tracking the intensity of the facial feedback effect. In the case of this particular research, the tracking of the intensity of the facial feedback effect would provide a possibility for testing whether there is a difference in the strength of ES effect on portrait shots lit by flat light and space-induced light that express different emotions (a different set of stimuli would have to be designed later for measuring the ToM).

A portrait lighting setup was chosen because it enables the sense of depth (separation of the human face itself and its background) to be recreated, and the different emotions to be evoked through facial feedback effect, the strength of which can be measured in terms of the ES effect.

For the emotional expression variable, three discrete emotional states that are reliably distinguishable for measuring positive and negative affective states via fEMG (van Boxtel 2010) were chosen: Happiness (Zygomaticus Major), Anger (Corrugator Supercilli) and Disgust (Levator Labii). (Figure 9) shows two examples of happiness. The duration of each video clip was $5 \mathrm{sec}$; the onset of the emotion on the actress's face occurred at the 2 nd second (the facial expression changed from neutral to one of the three aforementioned facial expressions).

\subsubsection{Measures}

In this pilot experiment, we used subjective measures to assess the emotional reactions, space perception and empathy.

For emotional valence and arousal, we used the Self-Assessment Manikin (SAM) (Bradley, Lang 1994; Lang 1980), a test widely used in emotion research, which consists of two nine-point pictorial scales. One scale serves to rate the valence or pleasantness of emotional feelings, and depicts nine manikins ranging horizontally from happy (or positive) to unhappy (or negative); the other scale serves to rate the arousal or excitement of emotional feelings, and depicts nine manikins whose expressions range from excited (or aroused) to calm (or relaxed). 

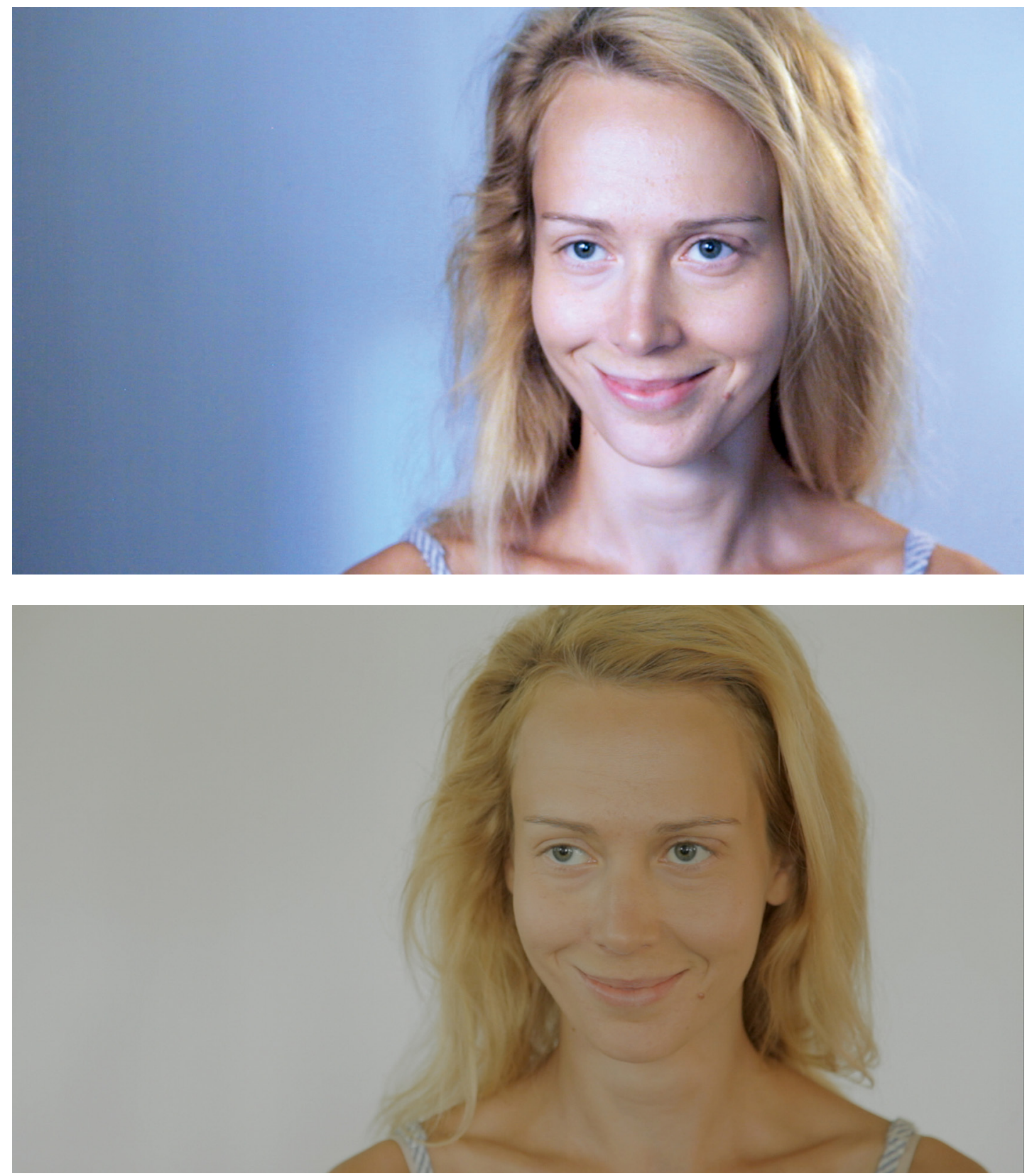

FIGURE 9. Snapshots of two video clips showing a happy facial expression in 'space-induced light' (A) and 'flat light' (B) conditions. Note that the light conditions were changed during the shooting and not created by post-processing of the same video clip. 
Space perception was based on an ITC-SOPI questionnaire (ITC-Sense of Presence Inventory) that assesses users' thoughts and feelings while and after being exposed to a media experience (Lessiter et al. 2001). ITC-SOPI assesses four factors - spatial presence, engagement, negative effects and ecological validity. We used two questions from the spatial presence factor, since this is the most directly related to the perception of space and is often referred to as a sense of 'being there' (Lombard, Ditton 1997). We used a 9-point interval-scale (one corresponding to strongly disagree and nine to strongly agree) and presented (in Estonian) the following questions (keeping the numbers of the ITC-SOPI questionnaire):

- $\quad$ (B34) I felt as though I was in the same space as the characters and/or objects.

- $\quad$ (B18) I had a sense of being in the scenes displayed.

Finally, we adapted two questions from Interpersonal Reactivity Index (IRI, Davis 1980), again with nine-point interval-scale and translated into Estonian:

- I have a tender, concerned feeling for this person.

- I felt indifferent how this person was feeling.

\subsubsection{Experiment design and procedure}

Two variables were the emotional facial expressions (three conditions: happiness, disgust and anger) and the two light conditions (space defining light vs. flat light). With three repetitions (three different actresses doing the same thing) for each of six conditions, a total of eighteen videos were shown.

The videos were shown in semianechoic chamber, with the participants sitting one metre from the 24" iMac screen. The videos were shown in random order, and after each video, the participants responded to six successively presented questions using a computer mouse.

\subsection{Results and discussion}

The IBM SPSS v20 software suite was used for statistical analyses. The alpha significance level was fixed at 0.05 for all statistical tests, and a Greenhouse-Geisser correction was used to compensate for unequal variances (Greenhouse, Geisser 1959). For multivariate analysis, Wilks' Lambda was used as the multivariate criterion. All variables were normally distributed according to the Kolmogorov-Smirnov test. Responses for two questions from both ITC-SOPI and IRI have been averaged to develop one spatial presence and empathy rating for each participant.

We ran 3 (emotional expressions) $\times 2$ (light conditions) $\times 3$ (different actresses) MANOVA including SAM valence and arousal, spatial presence and empathy ratings. As expected, there were no significant differences for any of the measures for the actresses (repetition) factor.

On multivariate level, the emotional expression factor reached significance $(p<0.02)$, demonstrating the validity of the employed emotional stimuli. For the univariate effects, the factor of emotional expressions again showed significant differences for all four measures $(p<0.05)$ (Figure 10).

Light conditions did not reach significance, except for the non-significant trend for valence ( $p=0.08)$, where the flat light condition shifted ratings towards a more negative evaluation. Importantly, there were significant interactions between emotional expressions factor and light conditions. Namely, the Disgust videos became significantly more negative in the flat light $(p<0.003)$ as compared to other two emotional expressions.

Neither spatial perception nor empathy ratings showed any significant results for the two factors. However, post-hoc pairwise comparison showed that the Happy video was significantly more favourable than other two conditions $(p<0.001)$ in terms of empathising.

To summarise, the results of this pilot experiment showed that there is a certain tendency for changes in valence with the increased rendering of the space in images that have been shot using cinematographic 


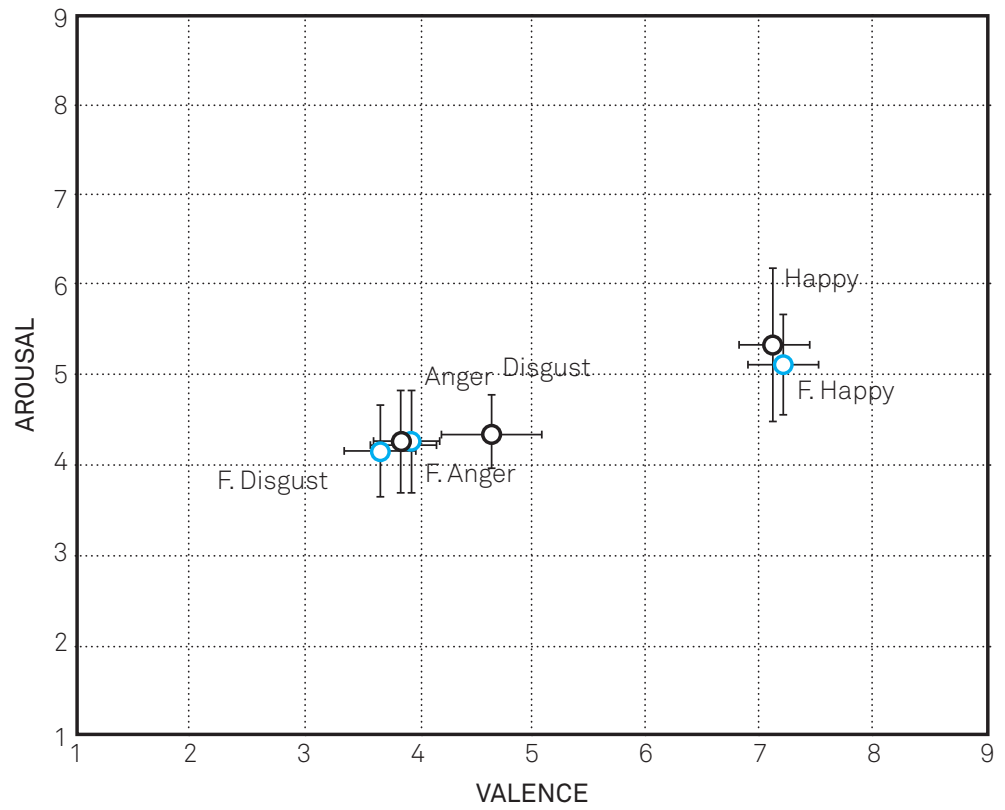

FIGURE 10. The subjective ratings for the arousal and valence of all six conditions (three emotional expressions and two light conditions). The red circles represent space-defining light and blue diamonds represent flat light condition. Error bars represent S.E.M. 
lighting canons that accentuate depth. Further experiments with a larger group and physiology (e.g. facial EMG) measurement need to be done in order to have more conclusive data for the two hypotheses - at the moment the first pilot experiment did not confirm either of the two. We also observed some individual preferences for one of the two types of light conditions - a further, more detailed questionnaire addressing space perception in participants will be considered for the main experiment. While three emotional expressions correspond well to the distinct facial expressions, it might be more difficult to empathise with anger or disgust feelings, so we might consider including videos depicting pain or frustration. Or, on the other hand, the viewer's self-reporting on negative feelings (anger and disgust) can indicate a negative reaction, while it is possible that the facial feedback effect will still be there when measured through fEMG showing the emergence of ES, although the viewers would not have distinct feelings towards the on-screen facial expressions of anger and disgust. As shown previously, the Embodied Simulation kind of empathy does not have to include a positive affect, but can cause direct mirroring of very negative states. This too needs to be measured and compared to the flat and space induced light variables.

\section{GENERAL CONCLUSIONS}

Could the reason for the lack of reciprocal knowledge sharing that could lead to new discoveries for both fields be the fact that today's researchers do not have enough interdisciplinary connections to practicing filmmakers? One of the benefits of including filmmaking practitioners in the research is the opportunity to become well-versed in the minute details of the field - details that have been accumulated as craft secrets by generations of cinematographers. One would hope that more of the same could occur in the other more specific fields of filmmaking - editing, sound design, production design, acting, directing etc. Based thereon, this article has attempted to connect cinematographic empathy with specific cinematographic tools that have been used throughout the history of film (and art). A lot of work can be done in this field and the connections between cognitive neuroscientists and cinematographers could result in breakthrough discoveries for other sciences as well.

To the best of the author's knowledge not much research has been done on cinematography, specifically in connection with the creation of empathy. This article has shown that there is already a growing body of research on empathy and film in general. There is also a lot of empiric experience that has been gained throughout film history, which seems to correspond to human cognitive and perceptual processes related to empathising. At the same time there are certain canons or 'trade secrets' in the cinematographic communities that are handed down through generations of cinematographers that deal mostly with the perception of space, and which seem to correspond to the specific needs of typical film genres for creating audience empathy. There are genres that need more immersion to achieve greater effects and others that need less immersion or even complete alienation. Cinematographers have developed tools for creating these effects. At the same time there is also a twofold division of empathy, one with more bodily and the other with more cognitive incidence. The article has shown that there is a possible correlation between the two types of cinematographic tools, the sense of space, and the two types of empathy.

\section{ACKNOWLEDGEMENTS}

I would like to thank Andero Uusberg, Helen Uusberg and Richard Naar for their help and input in developing the original design of the experiment. I would also like to thank Mati Mõttus for his help in preparing the experiment and collecting the data; Carlo Cubero for the supervision of the pilot experiment and Aleksander Väljamäe for the data analysis and the overall discussions regarding the manuscript. An important acknowledgement also goes to the actresses who participated in the creation of the experiment stimuli. 


\section{REFERENCES}

Andréasson, Per 2010. Emotional Empathy,

Facial Reactions, and Facial Feedback.

Acta Universitatis Upsaliensis. Digital Comprehensive Summaries of Uppsala Dissertations from the Faculty of Social Sciences 58. Uppsala: Uppsala Universitet. Berliner, Todd; Cohen, Dale J. 2011. 'The Illusion of Continuity: Active Perception and the Classical Editing System: - Journal of Film and Video 63, 1, 44-63.

Bickart, Kevin C.; Dickerson, Bradford C.; Barrett, Lisa Feldman 2014. 'The Amygdala as a Hub in Brain Networks That Support Social Life'. - Neuropsychologia 63, 235-248.

Bradley, Margaret M.; Lang, Peter J. 1994. 'Measuring Emotion: Self-Assessment Manikin and the Semantic Differential'. - Journal of Behavior Therapy and Experimental Psychiatry 25, 1, 49-59.

Brown, Blain 2013. Cinematography: Theory and Practice: Image Making for Cinematographers and Directors. New York, London: Focal Press.

Dalle Vacche, Angela 2009. 'Chiaroscuro:

Caravaggio, Bazin, Storaro'. - Senses of Cinema 53. http://sensesofcinema.com/2009/feature-articles/ chiaroscuro-caravaggio-bazin-storaro/ (1 November 2016).

Davis, Mark H. 1980. 'A Multidimensional Approach to Individual Differences in Empathy'. - JSAS Catalog of Selected Documents in Psychology 10, 85.

Ferber, Susanne; Humphrey, Keith G.; Vilis, Tutis 2005. 'Segregation and Persistence of Form in the Lateral Occipital Complex.' - Neuropsychologia 43, 1, 41-51.

Gallese, Vittorio 2009. 'Mirror Neurons, Embodied Simulation, and the Neural Basis of Social Identification: - Psychoanalytic Dialogues 19, 5, 519-536. Greenhouse, Samuel W.; Geisser, Seymour 1959. 'On Methods in the Analysis of Profile Data'. Psychometrika 24, 2, 95-112.

Han, Shihui; Jiang, Yi; Humphreys, Glyn W.; Zhou, Tiangang; Cai, Peng 2005. 'Distinct Neural Substrates for the Perception of Real and Virtual Visual Worlds'. Neuroimage 24, 3, 928-935.

Hardy, Robert 2015. 'A Primer on Practical Lights: How Practicals Can Make Your Cinematography Come to Life.' - No Film School, 2 July. http://nofilmschool. com/2015/07/practical-light-primer-how-practicallighting-bring-cinematography-to-life (3 November 2016).

Heider, Fritz; Simmel, Marianne 1944. 'An Experimental Study in Apparent Behavior'. - The American Journal of Psychology 57, 243-259.

Hillis, Argye E. 2014. 'Inability to Empathize: Brain Lesions That Disrupt Sharing and Understanding Another's Emotions'. - Brain 137, 4, 981-997. Hurlemann, René; Patin, Alexandra; Onur, Oezguer A.; Cohen, Michael X.; Baumgartner, Tobias; Metzler, Sarah; Dziobek, Isabel; Gallinat, Juergen; Wagner, Michael; Maier, Wolfgang; Kendrick, Keith M. 2010. 'Oxytocin Enhances Amygdala-Dependent, Socially Reinforced Learning and Emotional Empathy in Humans. - The Journal of Neuroscience 30, 14, 4999-5007.

Kilner, James M.; Paulignan, Y.; Blakemore,

Sarah-Jayne 2003. 'An Interference Effect of Observed Biological Movement on Action: - Current Biology 13, 6, 522-525.

Lang, Peter J. 1980. 'Behavioral Treatment and Bio-behavioral Assessment: Computer Applications'. Joseph B. Sidowski, James Harding Johnson, Thomas Arthur Williams (eds.), Technology in Mental Health Care Delivery Systems. Norwood, NJ:Ablex, 119-137. Lessiter, Jane; Freeman, Jonathan; Keogh, Edmund; Davidoff, Jules 2001. 'A Cross-Media Presence Questionnaire: The ITC-Sense of Presence Inventory. Presence: Teleoperators and Virtual Environments 10, 3, 282-297.
Lombard, Matthew; Ditton, Theresa B. 1997. 'At the Heart of It All:The Concept of Presence'. - Journal of Computer-mediated Communication 3, 2. http:// onlinelibrary.wiley.com/doi/10.1111/j.1083-6101.1997. tb00072.x/full (3 November 2016).

Marko, Bobby 2014. 'Helpful Ways for Cinematographers to Get a Grip on Lighting: Part 2'. - Provideo Coalition, 8 August. http://www.provideocoalition.com/ helpful-ways-for-cinematographers-to-get-a-grip-onlighting-part-2/\#sthash.7TfvrlJ0.dpuf (1 November 2016).

O'Hara, Ryan Patrick 2010. 'Why We Need Light: Shape \& Depth'- - Dvxuser, 18 August. http://www.dvxuser. com/V6/showthread.php?220459-RPO-s-Why-WeNeed-Light-Article-Series (3 November 2016).

Ousdal, Olga Therese; Specht, Karsten; Server, Andres; Andreassen, Ole A.; Dolan, Ray J.; Jensen, Jimmy 2014. 'The Human Amygdala Encodes Value and Space During Decision Making. - Neuroimage 101, 712-719.

Perani, D.; Fazio, F.; Borghese, N. A.; Tettamanti, M.; Ferrari, S.; Decety, J.; Gilardi, M. 2001. 'Different Brain Correlates for Watching Real and Virtual Hand Actions'. - Neuroimage 14, 3, 749-758.

Ramachandran, V. S.; Hirstein, William 1999.

'The Science of Art: A Neurological Theory of Aesthetic Experience.' - Journal of Consciousness Studies 6, 6-7, 15-51.

Raz, Gal; Hendler, Talma 2014. 'Forking Cinematic Paths to the Self: Neurocinematically Informed Model of Empathy in Motion Pictures. - Projections: The Journal for Movies and Mind 8, 2, 89-114.

Shutter Angle 2012. 'Creating Depth, Part 1: Introduction, DOF, Deep Staging, Resolution: - Shutter Angle, 24 November. http://www.shutterangle.com/2012/ creating-depth-dof-deep-staging-resolution/ (3 November 2016)

Stam, Robert; Burgoyne, Robert; Flitterman-Lewis, Sandy 1992. New Vocabularies in Film Semiotics. London, New York: Routledge.

Sutherland, Ainsley 2016. 'The Limits of Virtual Reality: Debugging the Empathy Machine'. - Docubase: MIT Open Documentary Lab. http://docubase.mit.edu/ lab/case-studies/the-limits-of-virtual-realitydebugging-the-empathy-machine/ (15 March 2016). van Boxtel, Anton 2010. 'Facial EMG as a Tool for Inferring Affective States.' - Proceedings of Measuring Behavior, 104-108.

Zaki, Jamil; Ochsner, Kevin N. 2012. 'The Neuroscience of Empathy: Progress, Pitfalls and Promise'. - Nature Neuroscience 15, 5, 675-680.

Zillmann, Dolf 2013. 'Empathy: Affect from Bearing Witness to the Emotions of Others'. - Jennings Bryant, Dolf Zillmann (eds.), Responding to the Screen: Reception and Reaction Processes. New York, London: Routledge, 135-168. 